\title{
Fibronectin-binding nanoparticles for intracellular targeting addressed by B. burgdorferi BBK32 protein fragments
}

\author{
Renate Ranka, $\mathrm{PhD}^{\mathrm{a}, \mathrm{b}, *}$, Ivars Petrovskis, $\mathrm{PhD}^{\mathrm{a}}$, Irina Sominskaya, $\mathrm{PhD}^{\mathrm{a}}$, Janis Bogans, $\mathrm{MSc}^{\mathrm{a}}$, \\ Ruta Bruvere, $\mathrm{PhD}^{\mathrm{a}}$, Inara Akopjana, $\mathrm{BSc}^{\mathrm{a}}$, Velta Ose, $\mathrm{PhD}^{\mathrm{a}}$, Irena Timofejeva, $\mathrm{MSc}^{\mathrm{a}}$, \\ Kalvis Brangulis, $\mathrm{MSc}^{\mathrm{a}}$, Pauls Pumpens, $\mathrm{PhD}^{\mathrm{a}}$, Viesturs Baumanis, $\mathrm{PhD}^{\mathrm{a}, \mathrm{b}}$ \\ ${ }^{a}$ Latvian Biomedical Research and Study Centre, Riga, Latvia \\ ${ }^{\mathrm{b}}$ University of Latvia, Riga, Latvia \\ Received 22 December 2011; accepted 2 May 2012
}

\begin{abstract}
Virus-like particles (VLPs) are created by the self-assembly of multiple copies of envelope and/or capsid proteins from many viruses, mimicking the conformation of a native virus. Such noninfectious nanostructures are mainly used as antigen-presenting platforms, especially in vaccine research; however, some of them recently were used as scaffolds in biotechnology to produce targeted nanoparticles for intracellular delivery. This study demonstrates the creation of fusion VLPs using hepatitis B core protein-based system maintaining a fibronectin-binding property from $B$. burgdorferi BBK32 protein, including the evidence of particles' transmission to BHK-21 target cells via caveolae/rafts endocythosis. These results make this construct to be an attractive model in development of HBc-based nanoparticles for cellular targeting applications and highlights the fragment of $B$. burgdorferi BBK32 as a novel cellular uptake-promoting peptide.
\end{abstract}

From the Clinical Editor: This paper discusses the nanotechnology-based application of self-assembling viral-like peptides (VLP-s) for targeted delivery using a hepatitis B core protein based system. Creating fusion VLPs may be an attractive model for cellular targeting applications. (C) 2013 Elsevier Inc. All rights reserved.

Key words: Fibronectin; Nanoparticles; B. burgdorferi

Various strategies in the design of nanoparticles (NPs) particles in the size range $1-1000 \mathrm{~nm}$ - aim to create new generations of drug-delivery vehicles, contrast agents, and diagnostic devices. ${ }^{1}$ One of the relevant potentials of the nanomedicines is their intracellular targeting possibility. Effective intracellular drug delivery is important for therapeutic agents that have specific molecular targets inside a cell as well as for drugs that undergo extensive efflux from the cell by the efflux transporters. Thus, the ability to penetrate inside cells bypassing lysosomal degradation is one of the key problems in the rational design of pharmaceutical nanocarriers. ${ }^{2}$ For this purpose, the surface of nanocarriers could be modified by certain internalizable ligands (e.g., folate, transferrin) or by cellpenetrating peptides, such as a trans-activating transcriptional

No conflict of interest was reported by the authors of this article.

This work was supported by grant of Latvian Council of Science, $\mathrm{Nr}$. 10.0029.3 and by ESF Project No. 1DP/1.1.2.0/09/APIA/VIAA/150.

*Corresponding author: Latvian Biomedical Research and Study Centre, Riga, LV-1067, Latvia.

E-mail address: renate_r@biomed.lu.lv (R. Ranka). activator (TaT), an integrin-binding peptide (RGD peptide) or polyArginine. ${ }^{3}$ This approach is receiving increasing attention over the last years because it is efficient for a range of cell types, and various endocytotic mechanisms can be engaged to facilitate the internalization of a carrier. ${ }^{2}$

Virus-like particles (VLPs) is a broad group of nanocarrier systems that exhibit great potential in biomedicine research, including targeted drug delivery. ${ }^{4}$ VLPs are self-assembling noninfectious supramolecular structures that have been produced from structural proteins of a wide variety of virus families. The unique features of VLPs are proper dimensions for nanoscale applications, size homogeneity, a large surface area-to-mass ratio, a symmetric macromolecular organization, biodegradability, biocompatibility and ease of production/ purification. ${ }^{5}$ In addition, a number of targeting molecules and peptides can be displayed in a biologically functional form on their capsid surface using chemical or genetic means. ${ }^{1}$ However, the issues of potential toxicity, biodistribution, clearance rates, stability, and immunogenicity are not fully addressed for these novel molecular devices for in vivo biomedical applications. 
A

BF 130-166

\begin{tabular}{|c|c|c|}
\hline HBC $1-78$ & PGS TQGSLNSLSGESGELEEPIESNEIDLTIDSDLRPKS D & HBC 79-183 \\
\hline
\end{tabular}

BF 160-175

\begin{tabular}{|c|c|c|}
\hline HBC 1-78 & PGS DLRPKSSLQGIAGSN SSD & HBC 79-183 \\
\hline
\end{tabular}

BF 153-175

BBK32: $160-175$

\begin{tabular}{|l|l|l|}
\hline HBc $1-78$ & PGS IDLTIDSDLRPKSSLQGIAGSN SSD & HBc 79-183 \\
\hline
\end{tabular}

BBK32: 153-175

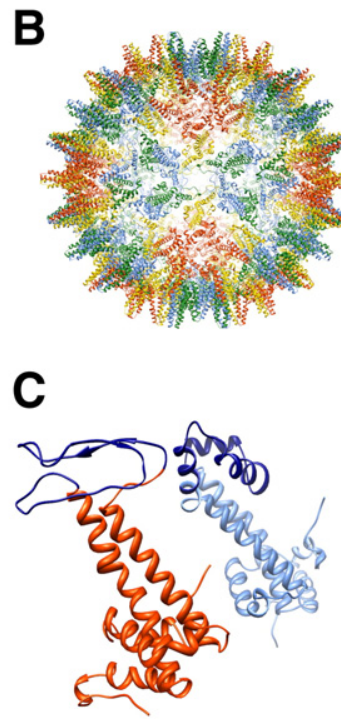

BF 130-166

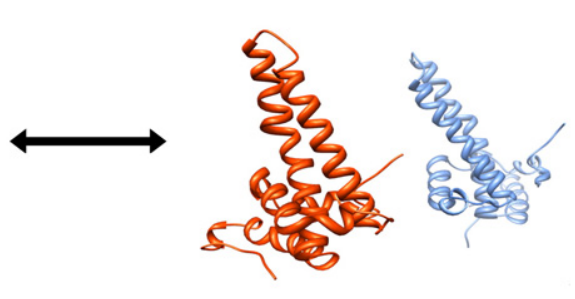

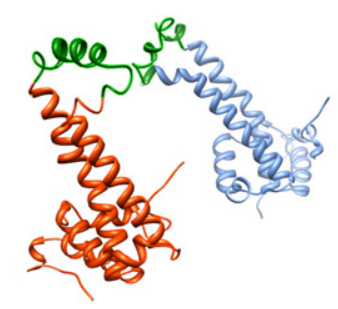

BF 160-175

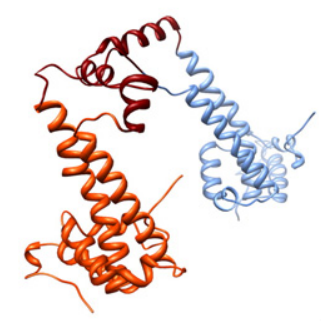

BF 153-175

Figure 1. (A) Schematic presentation of the fusion HBc/BBK32 constructs used in this study. BBK32 fragments were inserted in MIR of HBc protein. Gray boxes: HBc fragments. White boxes: BBK32 fragments. Abbreviations of grey color represents linkers (amino acids GS and SS), and amino acids, added due to introduction of the BamHI restriction site (amino acids P and D). (B) Three-dimensional prediction of the HBc VLP and individual HBc protein chains. (C) Three-dimensional prediction of the individual $\mathrm{HBc}$ protein chains carrying different fragments of the BBK32 protein. Predictions were performed on the basis of the x-ray structure of the $\mathrm{HBc}$, genotype $\mathrm{A},{ }^{7}$ by a comparative modeling program $3 \mathrm{D}-\mathrm{JIGSAW}$ (http://bmm.cancerresearchuk.org/3djigsaw/) ${ }^{30}$ and presented by Chimera software. ${ }^{31}$ Chains A (orange red) and D (cornflower blue) of the HBc asymmetric tetramer unit are presented, but $\mathrm{B}$ and $\mathrm{C}$ chains are omitted for visual clarity. Three BBK32 protein insertions are shown by blue, green and red.

Hepatitis B virus core $(\mathrm{HBc})$ is a $183-\mathrm{aa}, 21 \mathrm{kDa}$ protein which self-assembles to form $\sim 35$-nm particles that comprise the virion nucleocapsid. Dimer clustering of two $\mathrm{HBc}$ monomers produces spikes on the surface of the capsid, and the loop region located on top of these spikes (residues $78-83$ ) is the major target of the humoral response, i.e., major immunodominant region (MIR). ${ }^{6,7}$ Generally HBc-based VLPs have been used as an epitope presentation system in vaccine design and antigen presentation studies. ${ }^{8-11}$ On the other hand, the ability of HBc to incorporate peptides of different origin may be useful in the development of NPs for different application. ${ }^{12}$ For instance, packaging of enzymatically active nuclease, green fluorescent protein, RNA, and siRNA into the interior of recombinant hepatitis B virus capsids was demonstrated. ${ }^{13-16}$ Moreover, it was proposed that the inner VLP lumen may be modulated by $\mathrm{C}$ terminal domain modifications; the authentic arginine-rich $\mathrm{C}$ terminal domain packages RNA but may be replaced, within limits by heterologous moieties that could act as cargo or provide specific functions. ${ }^{17}$ However, transport of such particles in extracellular space and their uptake by different cells is of crucial significance for expression of their desired biological activity, so that it is necessary to design the appropriate targeting structures on the surface of VLPs to enhance cellular internalization.

Fibronectin (FN) is a common constituent of the extracellular matrix with important functions such as structural support and signaling for cell survival, migration, contractility, and differentiation. ${ }^{18} \mathrm{FN}$ is a large glycosylated mosaic protein composed of multiple copies of three types of modules: FNI, FNII, and FNIII. These modules comprise several functional domains that mediate interactions with cell-surface receptors (integrin-binding tripeptide, Arg-Gly-Asp [RGD] peptide), other ECM components (e.g., two heparin-binding domains, the 
Table 1

Oligonucleotide primers designed for amplification of BBK32 gene fragments

\begin{tabular}{lll}
\hline Construct & Forward primer & Reverse primer \\
\hline BF130-166 & 5'-CCGGATCCTGGATCAACTCAAGGAAGTTTA & 5'-TTGGATCCGAACTCTTTGGCCT \\
BF160-175 & 5'-ACCGGATCCTGGATCAGATTTACGTCCAAAGAG & 5'-TTGGATCCGAAGAGTTTGATCCTGC \\
BF153-175 & 5'-ACCGGATCCTGGATCAATTGATCTTACTATAG & 5'-TTGGATCCGAAGAGTTTGATCCTGC \\
\hline
\end{tabular}

collagen-binding domain [gelatin-binding domain, GBD], and two fibrin-binding sites), and FN itself. FN is secreted by different cell types as a disulfide-bonded, soluble inactive dimer that is subsequently assembled into an insoluble fibrillar network. ${ }^{19} \mathrm{FN}$ acts as a ligand for bacterial and viral adherence to host cells and plays a role in modulating the cellular entry of retroviral vector particles. $^{20,21}$ In addition, a number of studies suggested an active role of $\mathrm{FN}$ adsorption in cellular uptake of nonviral nanocarriers (for review, see Adler et al. ${ }^{22}$

A large amount of bacterial FN-binding protein has been identified so far; among them is BBK32, a $47 \mathrm{kDa}$ surfaceexposed lipoprotein and FN-binding adhesin of Borrelia burgdorferi (B. burgdorferi). ${ }^{23,24}$ BBK32 contains multiple FN-binding motifs located to an extended intrinsically disordered segment. $^{25}$ It was shown that the region of BBK32 comprising amino acids 147 - 205 binds to the N-terminal FNI and FNIII modules, but residues 120 - 147 of BBK32 protein are considered similar to the FN-binding region of $\mathrm{SfbI}$ protein from S. pyogenes that had been shown to bind to the GBD of FN. ${ }^{26-28}$

Here we present the construction of HBc-based chimeric VLPs with FN-binding property by insertion of $B$. burgdorferi BBK32 fragments into the MIR region of capsid. The evidence of intracellular internalization of these VLPs into nonphagocytic cells through caveolae-mediated endocytosis makes this construct an attractive model in development of HBc-based NPs for medically relevant cellular targeting applications and highlights the $130-166$ aa fragment of B. burgdorferi BBK32 as a novel cellular uptake-promoting peptide.

\section{Methods}

Construction of plasmids and prediction of structure of chimeric $\mathrm{HBc} / \mathrm{BBK} 32 \mathrm{VLPs}$

The construction design of fusion VLPs in this study involved the insertion of a foreign sequence into the MIR of $\mathrm{HBc}$ protein (Figure 1, A). The cloning vector $\mathrm{pHBc}$ encoding the full-length (amino acids 1-183) of HBc protein, subtype ayw, with inserted BamHI restriction site at MIR was constructed by M. Mihailova like those described in another study. ${ }^{29}$

Three-dimensional predictions of the reconstructed $\mathrm{HBc}$ structures were performed on the basis of the x-ray structure of the $\mathrm{HBc}$, genotype $\mathrm{A},{ }^{7}$ by a comparative modeling program $3 \mathrm{D}$ JIGSAW (http://bmm.cancerresearchuk.org/3 djigsaw/) ${ }^{30}$ and presented by Chimera software (Figure $1, B, C$ ). ${ }^{31}$

The fragments of BBK32 gene-encoding amino acids 130 to 166,160 to 175 , and 153 to 175 (BF130-166, BF160-175, and BF153-175 constructs, respectively) were amplified by PCR from B. burgdorferi sensu stricto isolate B31 DNA sample. The primers used to amplify these segments of BBK 32 are listed in
Table 1. A linker of two amino acids (GS) was added to the Nterminal end of the insert, and a serine $(\mathrm{S})$ was added to the $\mathrm{C}$ terminal end of insert. (The exception was BF130-166 construct, where both $\mathrm{C}$-end serines were of $B B K 32$ origin). BamHI restriction site was incorporated into both ends of inserts; the final peptide sequences are shown in Figure 1, $A$. The amplification products were digested with $B a m H$ I restriction endonuclease (Fermentas, Vilnius, Lithuania), purified using QIAEX II Gel Extraction Kit (Qiagen, Hilden, Germany), ligated into the linearized cloning vector, and competent $E$. coli RR1 ( $\mathrm{F}^{-}$leuB6 proA2 thi-1 araC14 lacY1 galK2 xyl-5 mtl-1 rpsL20 ( $\left.\mathrm{Str}^{\mathrm{r}}\right)$ glnV44 $\Delta$ (mcrC-mrr) cells were transformed with plasmid. The recombinant plasmids were isolated from positive clones by standard techniques, and correct constructs were verified by sequencing analysis.

\section{Expression and purification of recombinant VLPS}

Recombinant proteins were expressed in E. coli strain K802 by standard techniques and purified by the combination of ion exchange chromatography (IEC) on Q-HP Sepharose (GE Healthcare, Helsinki, Finland) and size-exclusion chromatography (SEC) on Sepharose 4FF (GE Healthcare) column methods (Supplementary Materials Sections S1 and S2). Protein samples were analyzed on a SDS-PAGE gel and by western blotting with mouse monoclonal anti-HBc $13 \mathrm{C} 9$ antibodies, ${ }^{32}$ as well as by double radial immunodiffusion according to Ouchterlony using polyclonal rabbit anti-HBc antibodies (Dako, Glostrup, Denmark).

\section{Electron microscopy}

The purified fusion VLPs in suspension were adsorbed on carbon-formvar-coated copper grids and negatively stained with $1 \%$ uranyl acetate aqueous solution. The grids were examined with a JEM-100C electron microscope (JEOL Ltd., Tokyo, Japan) at an accelerating voltage of $100 \mathrm{kV}$.

\section{Evaluation of the FN-binding activity by ligand affinity blot}

For the qualitative dot blot assay chimeric HBc/BBK32 VLPs were spotted onto the nitrocellulose membrane and probed with FN from human plasma followed by incubation with anti-FN antibodies (Supplementary Materials Section S3). As a control, recombinant full-length $(\mathrm{HBc})$ core particles expressed in E. coli were used.

\section{Quantitative FN-binding assays on microtiter wells}

Microtiter wells were coated with purified recombinant BF130-166 or $\mathrm{HBc}$ proteins and probed with increasing concentrations $(0-10 \mu \mathrm{g} / \mathrm{mL})$ of $\mathrm{FN}$ from human plasma followed by incubation with anti-FN antibodies. For experiments analyzing the role of gelatin- and heparin- binding domains of 
FN in the interactions between BF130-166 and FN, competitive binding analyses were performed by incubating $0.5 \mu \mathrm{g} / \mathrm{mL}$ of $\mathrm{FN}$ to immobilized $\mathrm{BF} 130-166$ and $\mathrm{HBc}$ proteins in the presence of gelatin or heparin at four competitor/FN molar ratios $(0.0 / 1,0.25 / 1,0.5 / 1$ and $1 / 1)$ (Supplementary Materials Section S4).

\section{Cellular entry assay}

For the cellular entry assays, BF130-166 VLPs were added to the medium of baby hamster kidney BHK-21 cells to final concentration $5 \mu \mathrm{g} / \mathrm{mL}$, and cells were incubated under different conditions, each experiment was repeated three times. (i) To investigate time-dependent uptake, cells were incubated with VLPs for $5,15,30,45$ and 60 minutes at $37^{\circ} \mathrm{C}$. (ii) To study the uptake in the presence of gelatin, cells were pretreated with gelatin $(5 \mu \mathrm{g} / \mathrm{mL}$ or $20 \mu \mathrm{g} / \mathrm{mL})$ for 15 minutes and incubated with VLPs in the presence of gelatin for 45 minutes at $37^{\circ} \mathrm{C}$. (iii) To study the effect of sodium azide treatment, cells were incubated with VLPs in the presence of $100 \mathrm{mM}$ sodium azide in the medium for 45 minutes at $37^{\circ} \mathrm{C}$. (iii) To study the effect of chlorpromazine $(5 \mu \mathrm{g} / \mathrm{mL})$ and filipin $(5 \mu \mathrm{g} / \mathrm{mL})$ treatment, cells were pretreated with drug for 30 minutes $^{33}$ and incubated with VLPs in the presence of drug for 45 minutes at $37^{\circ} \mathrm{C}$.

After incubation supernatants were removed and the cells were washed once with ice-cold PBS, twice with an ice-cold mild acidic wash buffer to remove external particles. The localization of VLPs and FN in cellular space was evaluated by fluorescent double immunostaining method (Supplementary Materials Section S5). For comparison, $\mathrm{HBc}$ particles were used. Samples were examined with a laser confocal fluorescence microscope Leica TCS SP2SE. As a negative control cells incubated without VLPs were used.

\section{Results}

\section{Synthesis and purification of fusion VLPS}

Three recombinant plasmids encoding different fusion $\mathrm{HBc} /$ BBK32 proteins were successfully constructed according to the sequencing analysis of plasmid DNA (data not shown). Figure 1, $C$ presents three-dimensional predictive reconstructions of the chimeric $\mathrm{HBc}$ protein chains, which are exposing the appropriate BBK32 fragments on the tips of the HBc spikes. The BBK32 structure 130-166 seems to be the most flexible, because predictions for the four $\mathrm{HBc}$ chains vary from the mostly $\alpha$ helical (chain D) to the $\beta$-sheet-forming (chains A, B, and C). The 153-175 and 160-175 insertions are predicted as mostly $\alpha-$ helice-forming for all four $\mathrm{HBc}$ chain variants.

Obtained plasmids were transformed into $E$. coli and recombinant proteins were expressed. SDS-PAGE and western blot analysis indicated the expression of soluble recombinant proteins of expected molecular weight, the levels of expression were sufficient for the further purification procedures (Figure 2, $A$ and $2, B)$. Expressed recombinant $\mathrm{HBc} / \mathrm{BBK} 32$ proteins were purified from E. coli; the purity of the isolated proteins according to Coomassie blue staining of the SDS-PAGE gel was $95 \%$ (Figure 2, A). The final yields of the recombinant proteins varied
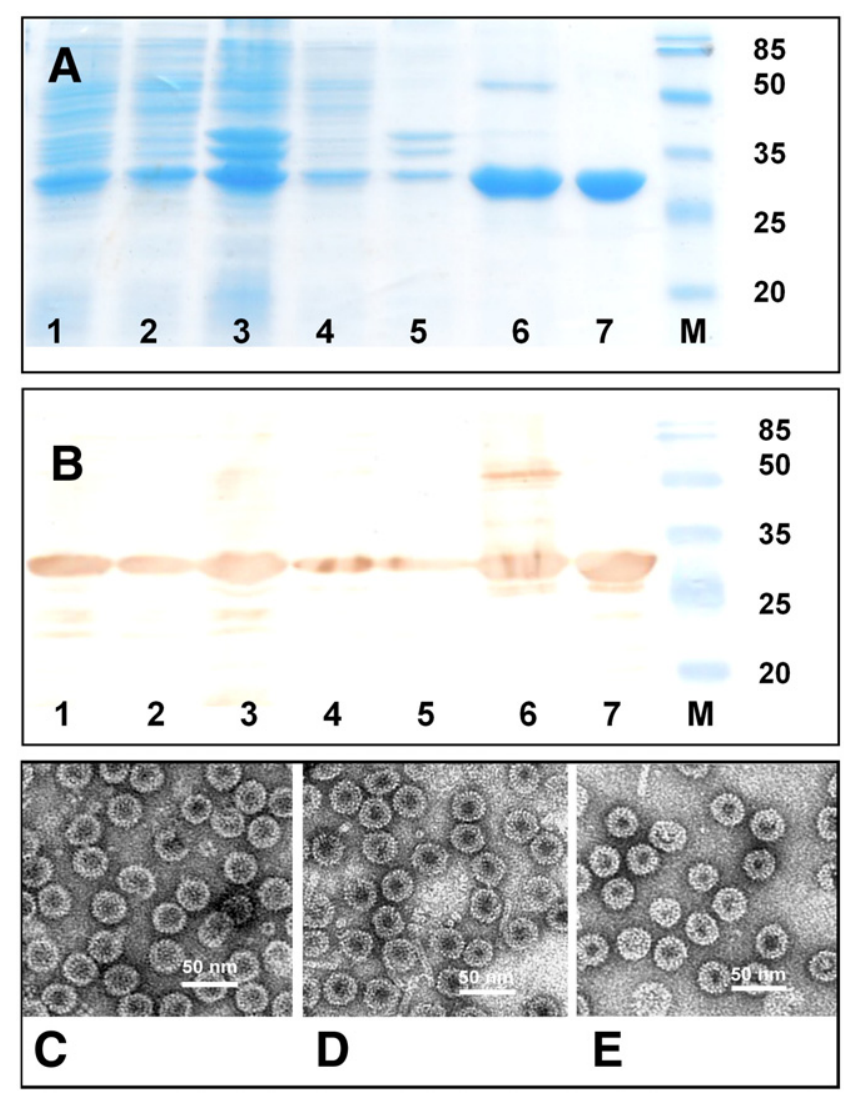

Figure 2. Expression, purification and morphologic analysis of fusion $\mathrm{HBc} /$ BBK32 VLPs. Fusion $\mathrm{HBc} / \mathrm{BBK} 32$ proteins were expressed in $E$. coli cells and purified by the combination of techniques. The expression levels of the proteins of interest were similar; the purity of the isolated proteins according to Coomassie blue staining of the SDS-PAGE gel was $95 \%$. Spherical particles of uniform size were obtained from all three constructs. Construct BF130-166 is shown as an example. (A) SDS-PAGE of protein samples, Coomassie brilliant blue staining, (B) Western blot with mouse monoclonal anti-HBc 13C9 antibodies. Lane 1: lysate of E. coli cells, lane 2: $0.5 \mathrm{M}$ Urea supernatant, lane 3: $0.5 \mathrm{M}$ Urea cellular debris, lane 4: supernatant after precipitation with ammonium sulfate at $10 \%$ saturation, lane 5: precipitate after precipitation with ammonium sulfate at $10 \%$ saturation, lane 6: protein fraction purified by IEC on Q-HP Sepharose, lane 7: protein fraction purified by SEC on Sepharose $4 \mathrm{FF}$ column, lane M: prestained protein molecular weight marker in $\mathrm{kDa}$. $(\mathbf{C}, \mathbf{D}, \mathbf{E})$ : Negative-staining electron microscopy images of the purified BF130-166 (C), BF160-175 (D) and BF 153-175 (E) protein. Scale bars (in white) are $50 \mathrm{~nm}$ as indicated.

between $5 \mathrm{mg}$ and $8 \mathrm{mg}$ per $1 \mathrm{~g}$ of $E$. coli cells (data not shown). Further examination of these preparations by electron microscopy revealed the presence of well-assembled particles of similar size (approximately $30 \mathrm{~nm}-35 \mathrm{~nm}$ ) and shape for all three constructs (Figure 2, C, D, E).

\section{FN-binding property of chimeric VLPS}

It was proposed that incorporation of B. burgdorferi BBK32 protein peptides harboring a FN-binding property into the hepatitis B core virus-like particles would allow us to create VLPs with enhanced FN-binding activity. The FN-binding property of obtained VLPs was evaluated by dot blot analysis by 


\section{A}
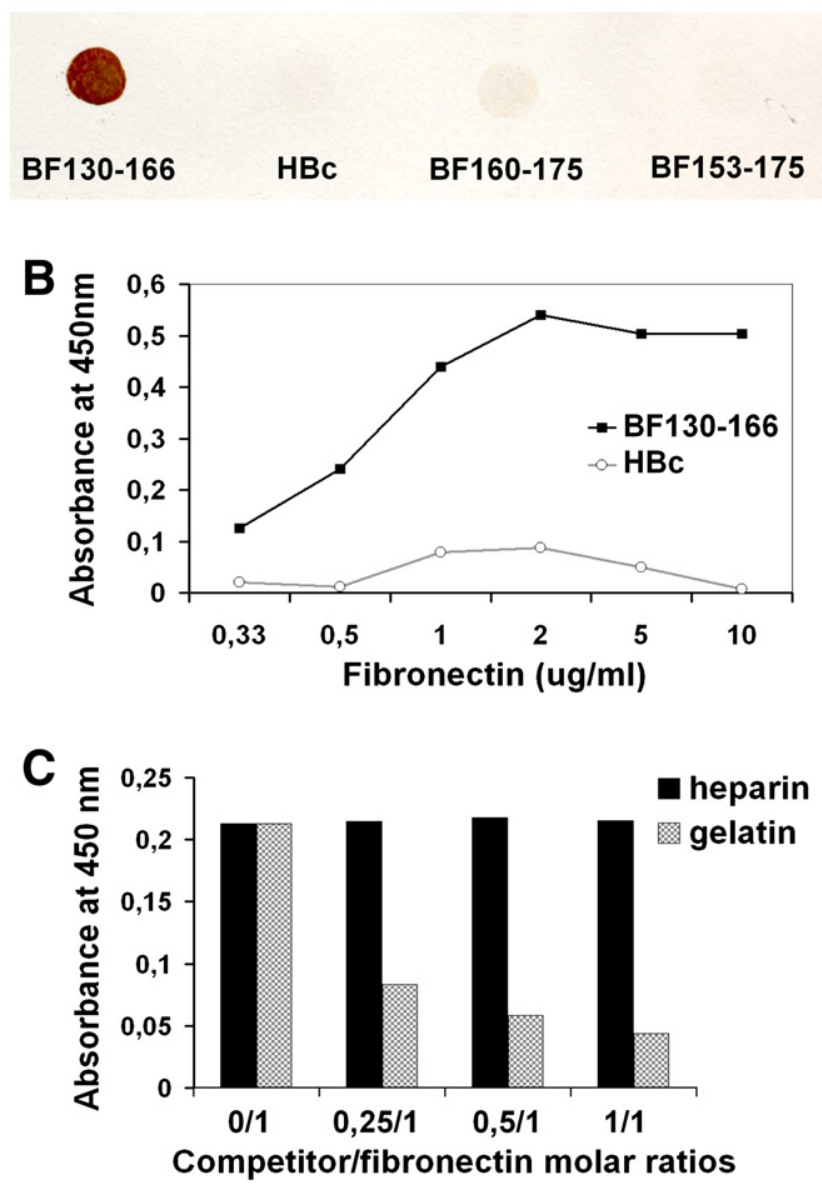

Figure 3. FN-binding properties of fusion HBc/BBK32 VLPs. (A) Dot blot analysis. Here, $1 \mu \mathrm{g}$ of purified VLPs was dotted on nitrocellulose membrane for ligand blotting with FN from human plasma. The VLPs designation is as follows: BF130-166, fusion HBc VLPs including amino acids 130 to 166 of $B$. burgdorferi BBK32 protein; HBc, recombinant VLPs containing unmodified hepatitis B core protein; BF160-175 and BF153-175, fusion HBc VLPs including amino acids 160 to 175 or 153 to 175 of B. burgdorferi BBK32 protein, respectively. Only the BF130-166 VLPs bind human FN efficiently. (B) Dose-dependent, saturable binding of human FN to BF130-166 protein. Binding of FN to immobilized BF130166 protein and $\mathrm{HBc}$ protein was analyzed by ELISA, with bound FN detected by anti FN antibodies. (C) Effects of gelatin and heparin on BF130-166 - FN interactions. Binding of human FN to immobilized BF130-166 protein in the presence of gelatin (gray bars) and heparin (black bars) was analyzed by ELISA, at gelatin or heparin/FN molar ratios of 0/1, $0.25 / 1,0.5 / 1$ and $1 / 1$. Bound FN was detected by anti-FN antibodies. Dosedependent inhibition of FN binding by gelatin was observed. As a negative control of the ELISA assays, corresponding amounts of FN were added to $5 \%$ milk-blocked wells, which did not contain any other immobilized protein. Values represent $\mathrm{FN}$ binding minus background readings for negative control wells.

use of a human FN as possible ligand, and recombinant fulllength HBc VLPs were used as a control. The results show that only BF130-166 VLPs have pronounced FN-binding activity (Figure 3,A). Very slight FN-binding activity was detected for the construct BF160-175. No FN-binding activity was detected for BF 153-175 construct and HBc VLPs. Reverse ligand affinity blot, in which FN, collagen, gelatin, and BSA were spotted on the membrane and probed with recombinant VLPs followed by incubation with polyclonal rabbit anti-HBcAg antibody, demonstrated similar results. No binding was observed for collagen/ gelatin or BSA (data not shown).

The ability of BF130-166 to bind FN was further evaluated by quantitative ELISA. Saturable, dose-dependent binding of FN to BF130-166 was observed (Figure 3, B). The binding of FN was specific for BF130-166 as the results for the recombinant insertfree $\mathrm{HBc}$ VLPs could not be considered as positive.

\section{Binding of BF130-166 to GBD of FN}

FN is a multifunctional molecule that interacts with a number of ligands via several distinct structural and functional domains. The BBK32 fragment of the BF130-166 construct contains putative GBD-binding motif ${ }^{28}$; therefore we next examined the potential role of this FN domain in BF130-166 interactions with FN. For this reason, FN binding by immobilized BF130-166 was examined by ELISA in the presence of increasing concentrations of gelatin, a known ligand for GBD. In a parallel assay, the adhesion of FN to immobilized BF130-166 was examined in the presence of increasing concentrations of heparin targeted to block the heparin-binding domains of FN. As shown in Figure 3, $C$, dose-dependent inhibition of FN binding by gelatin was observed. In contrast, the presence of heparin had no effect on FN - BF130-166 binding activity. These results indicated that recombinant BF130-166 VLPs interact with GBD of the FN molecule.

\section{Cell-permeability of fusion BF130-166 VLPS}

Because clear and strong FN-binding properties were observed only for the construct BF130-166, the possible internalization of these VLPs into BHK-21 cells was further studied by confocal laser scanning microscopy. Culture of BHK21 cells was inoculated by BF130-166 particles and the time course of internalization of VLPs was studied over 60 minutes' incubation time at $37^{\circ} \mathrm{C}$. For comparison, $\mathrm{HBc}$ particles were used. The localization of VLPs and FN in cellular space was evaluated by fluorescent double immunostaining method. Only green fluorescence $(\mathrm{FN})$ was observed in mock treated cells, as expected (Figure 4, Control).

In BF130-166 NP-treated cells, the VLPs appeared as nanosized fluorescent dots (red) mostly associated with cellular membranes after 5 minutes' incubation time. These VLPs were not removed from the cellular surface during the acid wash; thus we can suggest that this may reflect the initial cellular internalization stage. The staining pattern of VLPs was associated with the FN fluorescence (green); therefore, these results suggested co-localization of VLPs with FN in the cell line studied (Figure 4). Further analysis revealed enhancement of intracellular red signal in cell samples at 15 minutes' and 30 minutes' incubation time. In these images VLP- positive staining mostly accumulated into discrete foci resembled a typical endosomal or lysosomal punctuate pattern (Figure 4, 15', and 30 '). Subsequently the TRITC fluorescence signal became reduced and more dispersed as the time of incubation increased; only a few red fluorescent dots were observed in cells after 60 


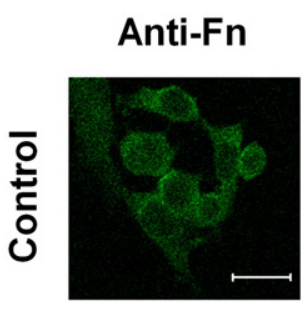

Anti-HBcAg Merge+DAPI

in
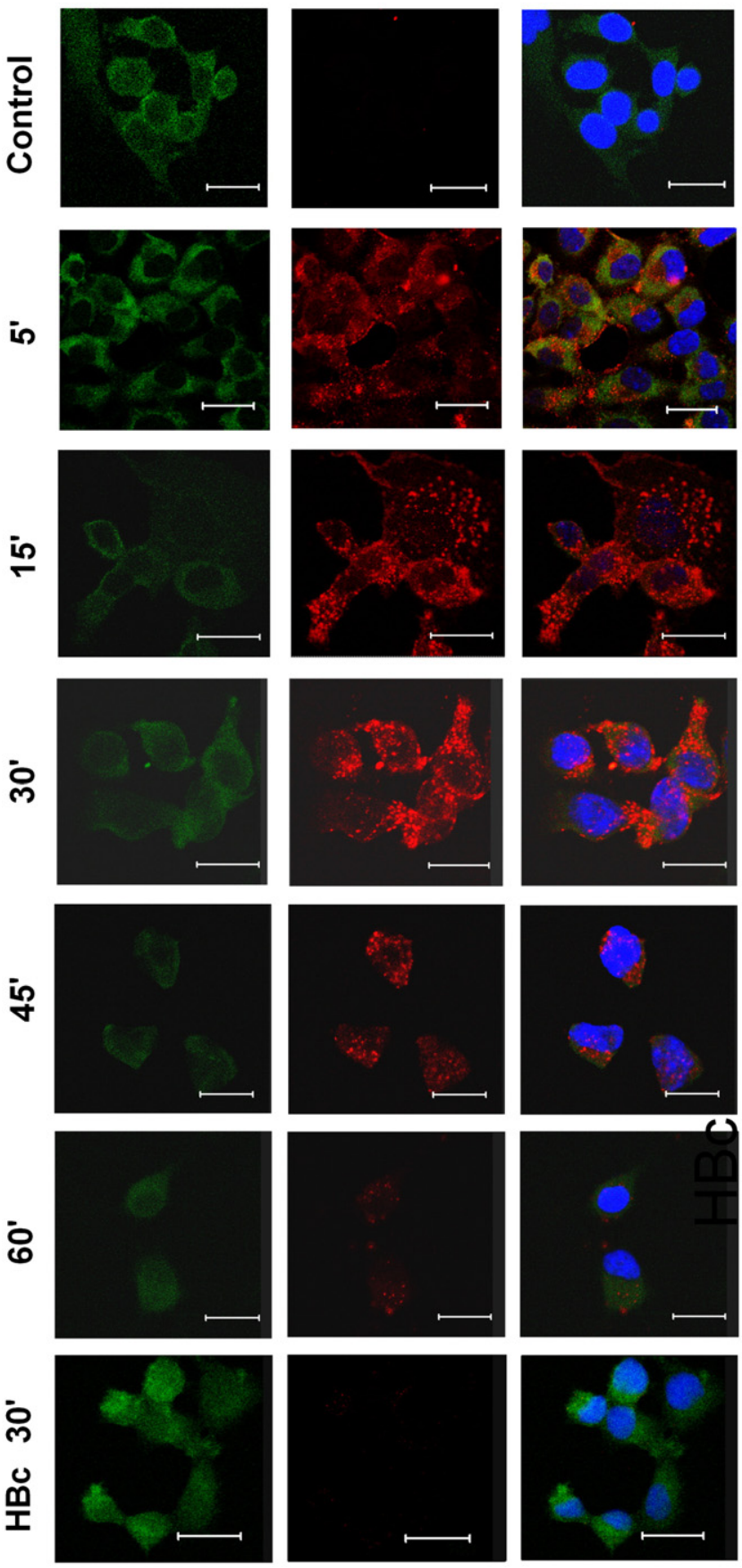

Figure 4. Fusion BF130-166 VLPs transmission to BHK-21 cells assessed by confocal microscopy. Culture of BHK-21 cells were inoculated by VLPs and the uptake of VLPs is depicted after 5, 15, 30, 45, and 60 min incubation time at $37^{\circ} \mathrm{C}$ following acid wash to remove external particles. Cells were stained with polyclonal rabbit anti-HBc antibody followed by TRITC-conjugated anti-rabbit IgG secondary antibody (red), and monoclonal anti-FN antibody followed by FITC-conjugated antimouse secondary antibody (green) in double immunostaining experiment. Cells' nuclei were stained with 4',6diamidino-2-phenylindole (DAPI, blue). No signal was observed in mocktreated cells (Control). No intracellular VLP-positive signal was observed for HBc VLPs. Scale bars (in white) are $20 \mu \mathrm{m}$ as indicated. minutes' incubation time (Figure 4, Anti-HBcAg, 60`). These results suggest a process of intracellular degradation of the NPs. In contrast, we did not observe pronounced changes in the green fluorescence pattern in cells over time (Figure 4, Anti-FN, images $\left.5^{`}-60^{\prime}\right)$. The intracellular localization of HBc VLPs was not observed (Figure 4, HBc 30').

\section{Cellular uptake of BF130-166 particles is inhibited by gelatin}

Given the separable nature of particle attachment and cell entry, we wanted to explore the possible role of binding to $\mathrm{FN}$ in the cellular uptake. For this purpose increasing concentrations of gelatin (5 and $20 \mu \mathrm{g} / \mathrm{mL}$ ) were added to BHK-21 cells 15 minutes prior to experiments and remained present during the incubation with VLPs. The result shows a decrease of VLPspositive signal in cells pretreated with $5 \mu \mathrm{g} / \mathrm{mL}$ gelatin and an absence of this staining in cells pretreated with $20 \mu \mathrm{g} / \mathrm{mL}$ gelatin (Figure 5, Anti-HBcAg, +gel. $5 \mu \mathrm{g} / \mathrm{mL}$ and + gel. $20 \mu \mathrm{g} / \mathrm{mL}$ ). This observation confirms that BF130-166 particles internalized the cells after binding to GBD of FN and verifies the specificity of binding and uptake.

\section{Mode of uptake of BF130-166 particles}

Next, we carried out a series of investigations on the uptake mechanism. Endocytosis can be inhibited with the depletion of cellular energy resources. The BHK-21 cells were incubated with BF130-166 in the presence of sodium azide, which inhibits active transport processes. Results show that the level of fluorescent intensity in the cytosol was reduced dramatically relative to cells cultured in standard conditions (Figure 5, Anti$\mathrm{HBcAg},+\mathrm{NaN}_{3}$ ). These results suggest that recombinant BF130-166 particles were internalized by endocytosis.

Further to inhibit clathrin-mediated and caveolin-mediated endocytosis, BHK-21 cells were treated with either $5 \mu \mathrm{g} / \mathrm{mL}$ chlorpromazine or $5 \mu \mathrm{g} / \mathrm{mL}$ filipin for 30 minutes, respectively. The results show that chlorpromazine treatment did not inhibit the entry of BF130-166; this observation suggests the clathrinindependent internalization. In contrast, filipin, a sterol-binding agent that disrupts caveolae and caveolae-like structures, strongly inhibit the entry of BF130-166, suggesting a filipin-sensitive pathway (Figure 5, Anti-HBcAg, +Chlorprom. and +Filipin). These observations provide evidence that FN-binding BF130-166 VLPs enter the cells via caveolae/raft-dependent endocytosis.

\section{Discussion}

VLPs, including HBc-based NPs, are emerging nanocarrier platforms that can be tailored at the genetic level. The surface modification of such particles with an appropriate peptide molecule is one of the possible strategies to influence the ability of the engineered VLPs to interact with cells and tissues at a molecular level and mediate the uptake by mammalian cells. To date, several studies have been performed aimed at developing HBc-based chimeric VLPs that could serve as potential nanovehicles to target various cells. ${ }^{16,34,35}$ In this study, we tested the possibility of exploiting the B. burgdorferi FN-binding 


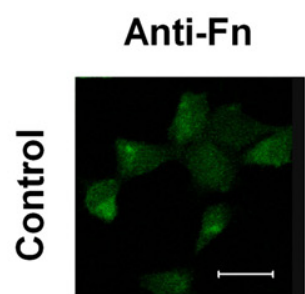

Anti-HBcAg Merge+DAPI
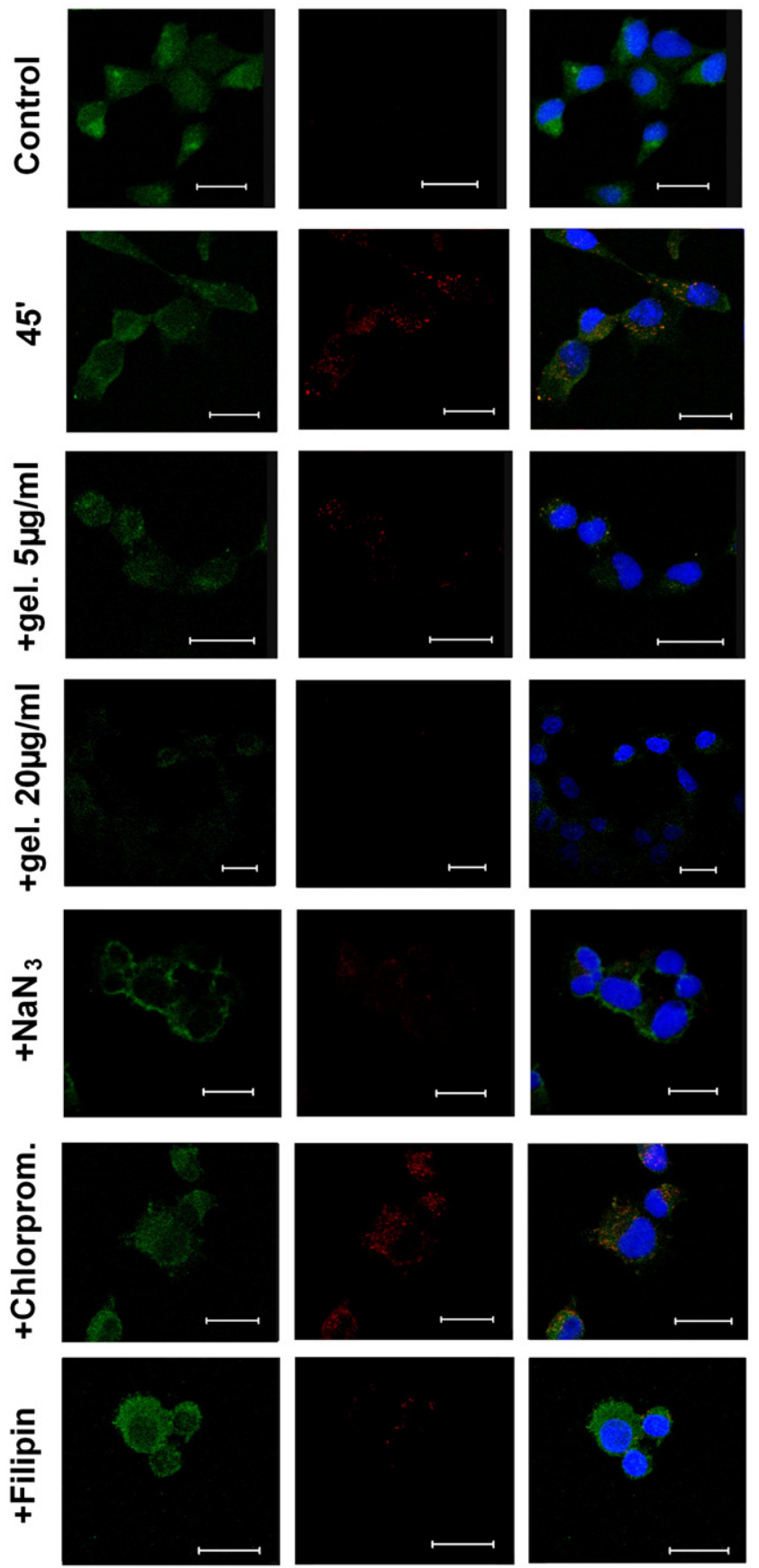

Figure 5. Fusion BF130-166 VLPs are endocytosed following attachment to FN. Culture of BHK-21 cells were inoculated by VLPs and the uptake of VLPs is depicted after 45 min incubation time at $37^{\circ} \mathrm{C}$ following acid wash to remove external particles. Cells were stained with polyclonal rabbit anti-HBc antibody followed by TRITC-conjugated anti-rabbit IgG secondary antibody (red), and monoclonal anti-FN antibody followed by FITC-conjugated antimouse secondary antibody (green) in double immunostaining experiment. Cells' nuclei were stained with 4',6diamidino-2-phenylindole (DAPI, blue). No signal was observed in mock-treated cells (Control). Pronounced red signal was observed in cells incubated with BF130-166 VLPs (45'). Similar signal was detected in cells pretreated with $5 \mu \mathrm{g} / \mathrm{mL}$ chlorpromazine and incubated with BF130166 VLPs in the presence of chlorpromazine (+Chlorprom.) Intracellular protein BBK32 for modification of $\mathrm{HBc}$ particles to create an FN-targeting system with subsequent transduction of target cells.

For this purpose, three fragments of B. burgdorferi FNbinding protein $\mathrm{BBK} 32$ were inserted in MIR of the $\mathrm{HBc}$ protein: amino acids 130 to 166,160 to 175 , and 153 to 175 (BF130-166, BF160-175, and BF153-175 constructs, respectively), and fusion proteins as VLPs were expressed in E. coli cells. HBc fusion proteins often maintain the self-assembling ability and display inserted foreign epitopes on the tips of particle spikes if they are inserted into the MIR. ${ }^{6}$ However, folding and capsid formations of the chimeric proteins are not always achieved easily, and the insert size and composition often are limitation factors in this process due to the conformational stress. Earlier it was shown that failure of some chimeric proteins to assemble into VLPs could be explained by the length of the insert; 120 amino acids are usually accepted as a maximum. On the other hand, Kratz et $\mathrm{al}^{36}$ reported successful insertion of the GFP protein $(238$ amino acids) in the $\mathrm{HBc}$; thus the structural importance of proper and independent folding of foreign sequences was clearly demonstrated. The potential role of different characteristics in the efficient assembly of VLPs was addressed in numerous studies, such as $\beta$-sheet forming properties, the distance between the $\mathrm{N}$ and the $\mathrm{C}$-terminus, and volume and hydrophobicity of the amino acids of the insert. However, the nature of the insert makes it often difficult to manipulate, and the design of successful constructs is still largely empirical. One of the most useful strategies to influence the flexibility of construct conformation is the addition of linkers. The linker size and composition was shown to have a strong effect on the VLPs' assembling property. ${ }^{6,37}$ To exploit these possibilities to overtake the assembly process, 41,21 , and 28 amino acids were inserted in $\mathrm{HBc}$ (BF130-166, BF 160-175, and BF153-175 constructs, respectively) including linkers. In addition, three-dimensional predictions of the reconstructed $\mathrm{HBc}$ structures were performed. The expression of all constructs in $E$. coli resulted in stable, soluble proteins that were effectively purified. Moreover, the electron microscopy data confirmed that all three $\mathrm{HBc} / \mathrm{BBK} 32$ fusion proteins assembled properly into symmetric particles indicating the successful design strategy (Figure 3). The average size of the particles calculated from the electron microscopy images was similar to the size of $\mathrm{HBc}$ VLPs, indicating that small-sized inserts did not have a great influence on the final dimensions of chimeric VLPs. Interestingly, recently the strategy of splitting the $\mathrm{HBc}$ protein inside the insertion loop (SplitCore) was offered to overcome almost accidental assembly process of chimeric VLPs. ${ }^{17}$

Another task of this study was to evaluate the FN-binding properties of obtained fusion VLPs. Different BBK32 peptides

VLP-positive signal was decreased in cells pretreated with $5 \mu \mathrm{g} / \mathrm{mL}$ gelatin and incubated with BF130-166 VLPs in the presence of gelatin for $45 \mathrm{~min}$ at $37^{\circ} \mathrm{C}(+\mathrm{gel} .5 \mu \mathrm{g} / \mathrm{mL})$. Intracellular VLP-positive signal was absent in cells pretreated with $20 \mu \mathrm{g} / \mathrm{mL}$ gelatin and incubated with BF130-166 VLPs in the presence of gelatin for $45 \mathrm{~min}$ at $37^{\circ} \mathrm{C}(+\mathrm{gel} .20 \mu \mathrm{g} / \mathrm{mL})$. The red signal was markedly reduced in cells pretreated and incubated in the presence of $100 \mathrm{mM}$ sodium azide $\left(+\mathrm{NaN}_{3}\right)$. The red signal was markedly reduced in cells pretreated and incubated in the presence of $5 \mu \mathrm{g} / \mathrm{mL}$ filipin (+filipin). Scale bars (in white) are $20 \mu \mathrm{m}$ as indicated. 
used in this study have been shown to bind different FN regions. $^{25-28}$ Our results show that only BF130-166 VLPs demonstrated clear, strong FN-binding ability. Moreover, we were able to show that this binding occurs via GBD of FN. Unfortunately, the nature of inserts in BF160-175 and BF153175 constructs seems to be insufficient to ensure stable ligand binding. It could be explained by the manner of binding of this BBK32 region to the $\mathrm{N}$-terminal modules of $\mathrm{FN}$ that require an antiparallel orientation of the binding partners with the peptide forming additional $\beta$-strands at the edge of the triple-stranded $\beta$ sheets of the two FNI modules (i.e., a "tandem-zipper" mechanism). ${ }^{26}$ The 153-175 and 160-175 insertions were predicted as $\alpha$-helice-forming for all four possible chain variants; therefore we could assume the lack of conformational compatibility in FN binding for these VLPs (Figure 1). However, induction of ordered aggregation of soluble FN and inhibition of endothelial cell proliferation similar to that of anastellin were shown for these BBK32 fragments, so there may be further interest in these constructs in the future. ${ }^{27}$

Recently, retroviral binding to FN was associated with increased infection of target cells and efficient gene transfer. ${ }^{21,38}$ Therefore, we tested the possible intracellular entry of the BF130-166 VLPs using BHK-21 as target cells. Indeed, the results of confocal microscopy analysis clearly demonstrated time-dependent particle uptake in BHK-21 cells. It was observed that entry process started with the attachment of VLPs to the cell surface followed by rapid internalization by energy dependent endocytosis. Initial particle co-localization with FN supported the idea that recombinant BF130-166 particles interact with FN molecules in the cellular microenvironment. Furthermore we were able to demonstrate the dose-dependant inhibition of the intracellular uptake by gelatin pretreatment. These results indicate that BF130-166 VLPs binding to the GBD of FN has an active role in the internalization process.

The relatively fast uptake was similar to the process observed for nonenveloped hepatitis $\mathrm{C}$ virus capsids in study by Katsarou et al. ${ }^{39}$ Clathrin-mediated endocytosis was reported as an entry pathway for these capsids as well as for HBc particles. ${ }^{39,40}$ In contrast, binding to $\mathrm{FN}$ has been previously reported to result in caveolae-dependent endocytosis of $\gamma$ - retrovirus vectors. ${ }^{20}$ Noteworthy, $\alpha 5 \beta 1$ integrin, a cellular receptor required for $\mathrm{FN}$ internalization, can be internalized by both clathrin-dependent and caveolar endocytosis. ${ }^{41}$ In the present study we have tested BF130-166 particles' entry in the presence of chlorpromazine and filipin, agents that block clathrin- and caveolae- dependent uptake, respectively. The results show that inhibition of clathrinmediated endocytosis had no effect on the uptake, but the disruption of membrane microdomains by the cholesterolchelating agent filipin completely opposes the internalization of fusion VLPs. Accordingly, these data suggest that BF130-166 NP endocytosis in BHK 21 cells occurs via a non-clathrinmediated mechanism and likely involves caveolae/lipid rafts. These findings may have significant implications because certain forms of caveolae-mediated endocytosis are thought to avoid the degradative lysosomal compartment. ${ }^{22}$

In conclusion, we were able to obtain FN-binding HBc-based VLPs with intracellular targeting properties. Although the potential applications of these NPs in humans may be limited due to possible immunogenicity and the abundance of FN in plasma, the evidence of particles' fast and efficient transmission to target cells opens the possibility for using this system as an intracellular delivery vector in vitro, as well as a model platform for investigation of multifunctional NPs with targeted features.

\section{Acknowledgments}

We thank Dr. Kaspars Tars for the suggestion of linkers used in recombinant constructs, Dr. Marija Mihailova for kindly providing the cloning vector, and Dr. Tatjana Kozlovska for helpful discussions.

\section{Appendix A. Supplementary data}

Supplementary data to this article can be found online at http://dx.doi.org/10.1016/j.nano.2012.05.003.

\section{References}

1. Cho K, Wang X, Nie S, Chen Z, Shin DM. Therapeutic nanoparticles for drug delivery in cancer. Clin Cancer Res 2008;14:1310-6.

2. Petros RA, DeSimone JM. Strategies in the design of nanoparticles for therapeutic applications. Nat Rev Drug Discov 2010;9:615-27.

3. Torchilin VP. Targeted pharmaceutical nanocarriers for cancer therapy and imaging. AAPS J 2007;9(2):E128-47.

4. Ma Y, Nolte R, Cornelissen J. Virus-based nanocarriers for drug delivery. Adv Drug Deliv Rev 2012;64:811-25.

5. Grasso S, Santi L. Viral nanoparticles as macromolecular devices for new therapeutic and pharmaceutical approaches. Int J Physiol Pathophysiol Pharmacol 2010;2:161-78.

6. Pumpens P, Grens E. HBV core particles as a carrier for B cell/T cell epitopes. Intervirology 2001;44:98-114.

7. Wynne SA, Crowther RA, Leslie AG. The crystal structure of the human hepatitis B virus capsid. Mol Cell 1999;3:771-80.

8. Ding FX, Xian X, Guo YJ, Liu Y, Wang Y, Yang F, et al. A preliminary study on the activation and antigen presentation of hepatitis $B$ virus core protein virus-like particle-pulsed bone marrow-derived dendritic cells. Mol Bio Syst 2010;6:2192-9.

9. Lee BO, Tucker A, Frelin L, Sallberg M, Jones J, Peters C, et al. Interaction of the hepatitis B core antigen and the innate immune system. $J$ Immunol 2009;182:6670-81.

10. Sun C, Ding FX, Wang F, He XW, He Y, Li ZS, et al. Screen of multifunctional monoclonal antibodies against hepatitis B core virus-like particles. Microbiol Immunol 2009;53:340-8.

11. Watts NR, Cardone G, Vethanayagam JG, Cheng N, Hultgren C, Stahl $\mathrm{SJ}$, et al. Non-canonical binding of an antibody resembling a naïve B cell receptor immunoglobulin to hepatitis B virus capsids. J Mol Biol 2008; 379:1119-29.

12. Garcea RL, Gissmann L. Virus-like particles as vaccines and vessels for the delivery of small molecules. Curr Opin Biotechnol 2004;15(6):513-7.

13. Beterams G, Böttcher B, Nassal M. Packaging of up to 240 subunits of a $17 \mathrm{kDa}$ nuclease into the interior of recombinant hepatitis $\mathrm{B}$ virus capsids. FEBS Lett 2000;481:169-76.

14. Lee KW, Tan WS. Recombinant hepatitis B virus core particles: association, dissociation and encapsidation of green fluorescent protein. J Virol Methods 2008;151(2):172-80.

15. Porterfield JZ, Dhason MS, Loeb DD, Nassal M, Stray SJ, Zlotnick A. Full-length hepatitis B virus core protein packages viral and heterologous RNA with similarly high levels of cooperativity. J Virol 2010; 84(14):7174-84. 
16. Choi KM, Choi SH, Jeon H, Kim IS, Ahn HJ. Chimeric capsid protein as a nanocarrier for siRNA delivery: stability and cellular uptake of encapsulated siRNA. ACS Nano 2011;5(11):8690-9.

17. Walker A, Skamel C, Nassal M. SplitCore: An exceptionally versatile viral nanoparticle for native whole protein display regardless of $3 \mathrm{D}$ structure. Sci Rep 2011;1 Article 5.

18. Pankov R, Yamada KM. Fibronectin at a glance. J Cell Sci 2002;115: 3861-3.

19. Mao Y, Schwarzbauer JE. Fibronectin fibrillogenesis, a cell-mediated matrix assembly process. Matrix Biol 2005;24:389-99.

20. Beer C, Pedersen L. Matrix fibronectin binds gammaretrovirus and assists in entry: new light on viral infections. J Virol 2007;81: 8247-57.

21. O'Neill LS, Skinner AM, Woodward JA, Kurre P. Entry kinetics and cell-cell transmission of surface-bound retroviral vector particles. $J$ Gene Med 2010;12:463-76.

22. Adler AF, Leong KW. Emerging links between surface nanotechnology and endocytosis: impact on nonviral gene delivery. Nano Today 2010; 5(6):553-69.

23. Henderson B, Nair S, Pallas J, Williams MA. Fibronectin: a multidomain host adhesin targeted by bacterial fibronectin-binding proteins. FEMS Microbiol Rev 2011;35:147-200.

24. Probert WS, Johnson BJ. Identification of a $47 \mathrm{kDa}$ fibronectin-binding protein expressed by Borrelia burgdorferi isolate B31. Mol Microbiol 1998;30:1003-15.

25. Kim JH, Singvall J, Schwarz-Linek U, Johnson BJ, Potts JR, Höök M. BBK32, a fibronectin binding MSCRAMM from Borrelia burgdorferi, contains a disordered region that undergoes a conformational change on ligand binding. J Biol Chem 2004;279:41706-14.

26. Raibaud S, Schwarz-Linek U, Kim JH, Jenkins HT, Baines ER, Gurusiddappa S, et al. Borrelia burgdorferi binds fibronectin through a tandem beta-zipper, a common mechanism of fibronectin binding in staphylococci, streptococci, and spirochetes. J Biol Chem 2005;280: 18803-9.

27. Prabhakaran S, Liang X, Skare JT, Potts JR, Höök M. A novel fibronectin binding motif in MSCRAMMs targets F3 modules. PLoS One 2009; 4:e5412.

28. Probert WS, Kim JH, Höök M, Johnson BJ. Mapping the ligand-binding region of Borrelia burgdorferi fibronectin-binding protein BBK32. Infect Immun 2001;69:4129-33.
29. Mihailova M, Boos M, Petrovskis I, Ose V, Skrastina D, Fiedler M, et al. Recombinant virus-like particles as a carrier of B- and T-cell epitopes of hepatitis C virus (HCV). Vaccine 2006;24:4369-77.

30. Bates PA, Kelley LA, MacCallum RM, Sternberg MJE. Enhancement of protein modelling by human intervention in applying the automatic programs 3D-JIGSAW and 3D-PSSM. Proteins 2001(Suppl 5):39-46.

31. Pettersen EF, Goddard TD, Huang CC, Couch GS, Greenblatt DM, Meng EC, et al. UCSF chimera - a visualization system for exploratory research and analysis. J Comput Chem 2004;25:1605-12.

32. Bichko V, Schodel F, Nassal M, Gren E, Berzinsh I, Borisova G, et al. Epitopes recognized by antibodies to denatured core protein of hepatitis B virus. Mol Immunol 1993;30:221-31.

33. Payne CK, Jones SA, Chen C, Zhuang X. Internalization and trafficking of cell surface proteoglycans and proteoglycan-binding ligands. Traffic 2007;8(4):389-401.

34. Brandenburg B, Stockl L, Gutzeit C, Roos M, Lupberger J, Schwartlander R, et al. A novel system for efficient gene transfer into primary human hepatocytes via cell-permeable hepatitis B virus-like particle. Hepatology 2005;42:1300-9.

35. Lee KW, Tey BT, Ho KL, Tan WS. Delivery of chimeric hepatitis B core particles into liver cells. J Appl Microbiol 2012;112:119-31.

36. Kratz PA, Bottcher B, Nassal M. Native display of complete foreign protein domains on the surface of hepatitis B virus capsids. Proc Natl Acad Sci USA 1999;96:1915-20.

37. Janssens ME, Geysen D, Broos K, De Goeyse I, Robbens J, Van Petegem F, et al. Folding properties of the hepatitis B core as a carrier protein for vaccination research. Amino Acids 2010;38:1617-26.

38. Sondergaard CS, Haldrup C, Beer C, Andersen B, Kohn DB, Pedersen L. Preloading potential of retroviral vectors is packaging cell clone dependent and centrifugation onto $\mathrm{CH}-296$ ensures highest transduction efficiency. Hum Gene Ther 2009;20:337-49.

39. Katsarou K, Lavdas AA, Tsitoura P, Serti E, Markoulatos P, Mavromara $\mathrm{P}$, et al. Endocytosis of hepatitis $\mathrm{C}$ virus non-enveloped capsid-like particles induces MAPK-ERK1/2 signaling events. Cell Mol Life Sci 2010;67:2491-506.

40. Cooper A, Shaul Y. Clathrin-mediated endocytosis and lysosomal cleavage of hepatitis B virus capsid-like core particles. J Biol Chem 2006;281:16563-9.

41. Caswell PT, Vadrevu S, Norman JC. Integrins: masters and slaves of endocytic transport. Nat Rev Mol Cell Biol 2009;10:843-53. 